\title{
A case of penile fracture caused by self manipulation to achieve detumescence
}

K. H.Palliyagiru, S. Suvendran, A.M. Abeygunasekera

Colombo South Teaching Hospital, Kalubowila. Sri Lanka

Key words : Penis; fracture; self manipulation.

A 47-year old man was admitted to the surgical emergency close to midnight with a painful swelling of his penis. He had been practicing self manipulation of the erect penis to achieve a rapid detumescence from early morning erections regularly for many years. He had forcibly bent the penis at mid shaft level on that particular morning. There had been a cracking sound and he experienced pain with gradual swelling of the penile shaft. Neither voiding difficulties nor haematuria was observed. Wet ice packs had been placed on the shaft to control the swelling and diclofenac sodium tablets were taken to control the pain. After 17 hours he admitted to the hospital as the swelling got worse and pain became severe despite analgesics.

Physical examination revealed a tender and swollen penis. We could not palpate a defect in the corpora/ shaft of the penis due to swelling and tenderness. A clinical diagnosis of acute penis due to penile fracture was made and consent was taken to explore the penis.

A circum-coronal incision was made after applying a tourniquet at the root of the penis, under general anaesthesia. The penis was degloved to expose the corpora cavernosa. There was a haematoma deep to the Buck's fascia over the proximal part of the right corpus cavernosum near the root of the penis (Figure 1). Evacuation of the blood clot revealed a rupture of the right corpus with a defect confirming the presumptive diagnosis of a penile fracture (Figure 2). The contused

Correspondence: A.M. Abeygunasekera

E-mail: amabey@sltnet.lk and irregular fascial edge was trimmed. The defect was repaired using 4/0 polyglactin sutures in two layers. There was no damage to the left corpus cavernosum. The corpus spongiosum with the urethra was intact. A circumcision was done and a urethral catheter was kept in situ for five days. Intravenous co-amoxiclav was given to avoid sepsis with oral non-steroidal analgesics for pain control.

The patient was experiencing good erections without pain or angulation when reviewed three weeks after surgery and had been so for the last two years.

\section{Discussion}

Acute pain and swelling of the penis is known as acute

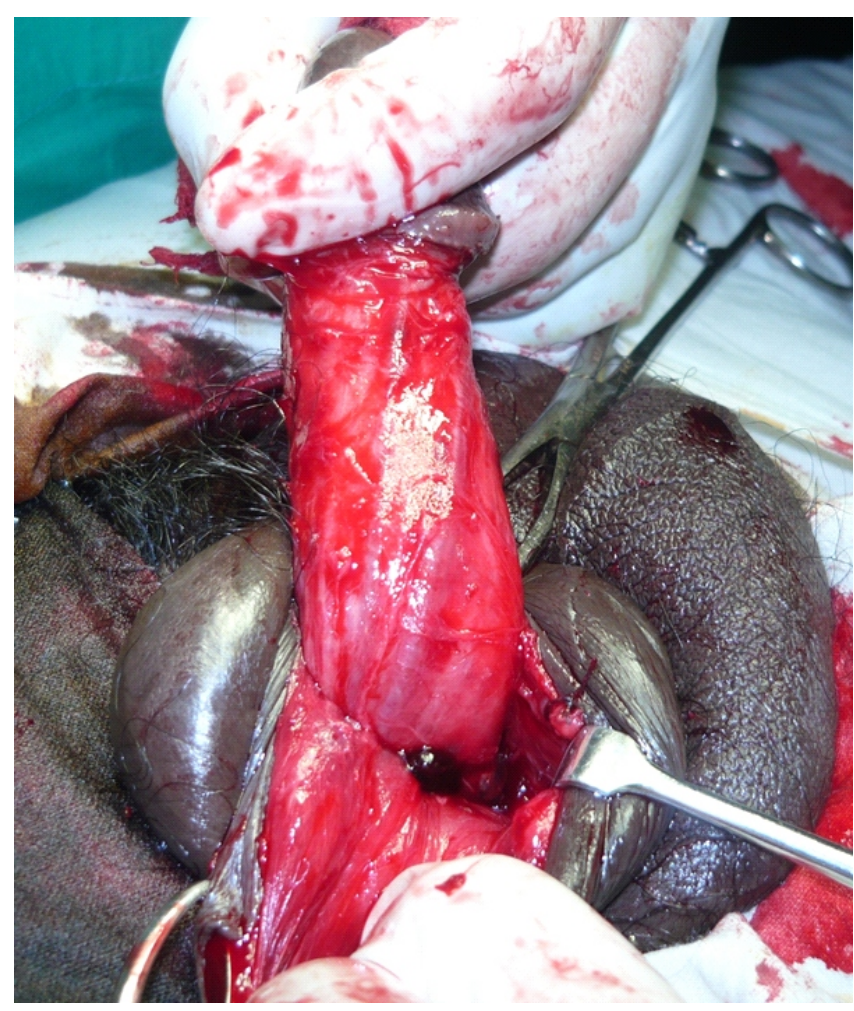

Figure 1. Haematoma at the fracture site of the corpus cavernosum 


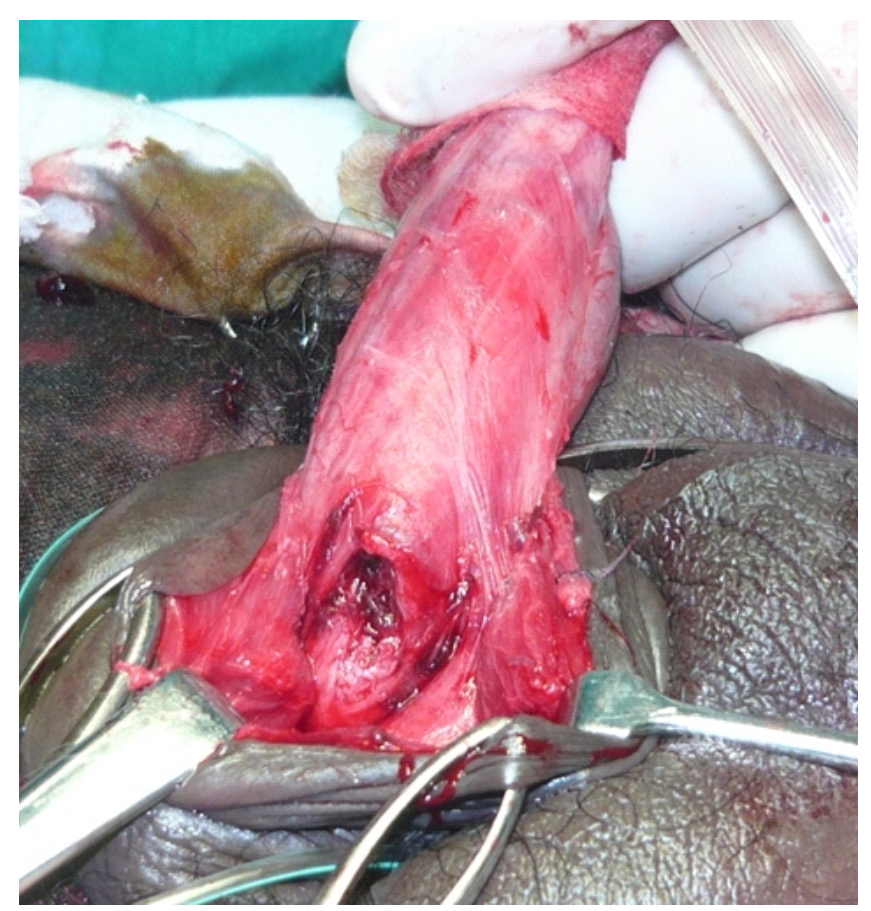

Figure 2. The defect in the corpus cavernosum

penis [1]. The usual causes of an acute penis are penile fracture or rupture of a dorsal vein of penis. Fracture is the commoner cause. Its incidence varies in different communities due to cultural differences. Certain cultures are associated with various manouveres related to the erect penis which can predispose to fracture.

Fracture of the penis is defined as disruption of the tunica albuginea and rupture of the corpus cavernosum. During erection the tunica albuginea thins from $2 \mathrm{~mm}$ to $0.25 \mathrm{~mm}$ and loses its elasticity which predisposes it to injury if abnormal forces are applied. It has been suggested that previous trauma could predispose to rupture at lower pressures than with a healthy tunica [2]. The mechanism of injury is usually a direct blunt force and sudden bending of the penis. It may occur during hasty sexual intercourse when the rigid penis slips from the vagina striking the partner's perineum or pubic bone. In Middle-eastern countries a common cause of penile fracture is self inflicted injury [3]. This is a practice termed taghaandan and occurs when the erect penis is bent to achieve detumescence. This practice has not been reported from a South Asian country. This patient was a Sri Lankan who belonged to the Sinhalese ethnic group where a similar practice has not been reported before.

While intercourse and self-manipulation account for most cases of penile fracture, there have been reports of fracture following a fall from bed with an erect penis, attempting to correct congenital chordee, disentangling the erect penis from a garment, forceful contact with the dashboard of a stopping car, masturbation or even unconscious nocturnal manipulation [4].

The events following penile fracture are characteristic and include a cracking or popping sound accompanied by pain, rapid detumescence, swelling and deviation of the penis to the side opposite of the injury. The defect at the fracture site may be palpable and represents a firm, mobile, tender mass where the penile skin can be rolled over the blood clot - 'rolling sign' [5]. The diagnosis is mainly clinical and investigations like cavernosography, ultrasonography and MRI are reserved for uncertain or atypical cases. In cases of suspected urethral injury a urethrogarm may become useful though not essential. Rupture of the deep dorsal vein is rare but the mechanism and clinical features are similar to penile fracture. Rupture of the superficial dorsal vein spreads through the subcutaneous tissues of the genitalia resulting in scrotal ecchymoses. Haematoma after rupture of the deep dorsal vein or penile fracture is deep to the Bucks fascia and thus remains within the penile shaft. Rarely urethral injury may occur with fracture of the penis. The reported incidence of associated urethral injury varies from 9 $20 \%$. Mostly the laceration is unilateral and involves one corpus carvernosum only, but in $2-10 \%$ of cases it may be bilateral.

Surgical intervention and repair of the injury produces better results than conservative measures like ice packs, compression bandages, fibrinolytics, anti-inflammatory agents and anti-androgens [6]. Early repair within 24 hours has been the recommendation traditionally for an 
optimum outcome [7]. However there are recent large studies with long-term follow-up which show that repair up to a week later produce equally good results [8]. Hence fracture of the penis may be considered a urological emergency anymore. Repair can be undertaken electively during the next seven days. Intravenous antibiotics and a urethral catheter for 24 to 72 hours are recommended. In the event of a repair of a urethral injury the urethral catheter should be retained for an extended period.

If untreated, patients after penile fracture may develop infection, urine extravasation, abscess formation and long-term complications like penile pain and deformity. Erectile dysfunction after penile fracture is uncommon. When rupture of the deep dorsal vein or superficial vein is the cause for an acute penis, ligation of the ruptured vein after evacuation of haematoma would suffice.

\section{References}

1. Karadenis T, Topaski M, Ariman A. Penile fracture: differential diagnosis, management and outcome. British Journal of Urology 1996; 77: 279-281

2. De Rosa AF, Giglio M, Cormignani G, Traumatic rupture of the corpora cavernosa: new pathophysiologic acquisitions. Urology 2001; 57:319-322
3. Zargooshi J. Penile fracture in Kermanshah, Iran: report of 172 cases. Journal of Urology 2000; 164: 364366

4. Hoag NA, Hennessey MD, So A. Penile fracture with bilateral corporeal rupture and complete urethral disruption: case report and literature review. Canadian Urology Association Journal 2011; 5: e23-e26

5. Narayansingh V, Raju GC. Fracture of penis. British Journal of Surgery 1985; 72:305-306

6. Morris SB, Miller MAW, Anson K. Management of penile fracture. Journal of Royal Society of Medicine $1998 ; 91: 427-428$

7. Abeygunasekera A. Fracture of the penis: a rare urological emergency. Galle Medical Journal 2000; 2: 18-19

8. El-Assmy A, el-Tholoth HS, Mohsen T, Ibrahiem I. Does timing of presentation of penile fracture affect outcome of surgical intervention? Urology2011; 77: 1389-1391

\section{Key learning points}

- Acute pain and swelling of the penis (acute penis) is commonly due to penile fracture or rupture of a dorsal vein of penis.

- The diagnosis is essentially clinical.

- Surgical intervention and repair of the injury produces better results and can be undertaken electively during the next seven days. 